\title{
Separation of Suspended Particles by Arrays of Obstacles in Microfluidic Devices
}

\author{
Zhigang $\mathrm{Li}^{*}$ and German Drazer ${ }^{\dagger}$ \\ Department of Chemical and Biomolecular Engineering, Johns Hopkins University, Baltimore, Maryland 21218, USA
}

(Received 30 June 2006; published 2 February 2007)

\begin{abstract}
The stochastic transport of suspended particles through a periodic pattern of obstacles in microfluidic devices is investigated by means of the Fokker-Planck equation and numerical simulations. Asymmetric arrays of obstacles have been shown to induce the continuous separation of DNA molecules, with particles of different size migrating in different directions within the microdevice (vector separation). We show that the separation of tracer particles only occurs in the presence of a permeating driving force with a nonzero normal component at the surface of the solid obstacles, and arises from differences in the local Peclet number of the particles. On the other hand, finite-size particles also exhibit nonzero, but small, migration angles in the case of nonpermeating fields. Monte Carlo simulations for different driving fields agree with the solutions to the Fokker-Planck equation.
\end{abstract}

DOI: 10.1103/PhysRevLett.98.050602

In recent years different strategies have been explored for the development of integrated microfluidic devices that provide rapid and efficient separation of suspended particles $[1,2]$. Particularly promising are novel methods that take advantage of Brownian motion to fractionate a mixture of colloidal particles. Several experiments and theoretical investigations have in fact shown that it is possible to rectify the Brownian motion of suspended macromolecules (DNA, in particular) by driving them through an asymmetric course of micrometer-scale obstacles [3-7]. Some of these devices have the additional advantage that different species migrate at different angles (vector separation), thus allowing for continuous fractionation (see Fig. 1 for a schematic representation of a microdevice). However, the initial explanation of this phenomena based on differences in the diffusivity of the suspended particles $[4,6,7]$ has been recently challenged by experimental observations and theoretical considerations [8-10]. Here we investigate the necessary conditions for particle separation to occur in spatially periodic systems by means of continuum models and Monte Carlo simulations. We show that there is no vector separation unless the driving force has a nonvanishing component normal to the solid obstacles. Moreover, we show that in the presence of a normal force, vector separation is based on differences in the local Peclet number of the particles. Therefore, differences in the diffusivity of the species would result in separation only if the local Peclet numbers is also different between species. On the other hand, we also show that particle size effects may induce differentiated migration even in the absence of forces normal to the obstacles.

The stochastic transport of suspended particles can be described by the Fokker-Planck equation for the probability density $P(\mathbf{x}, t)$,

$$
\frac{\partial}{\partial t} P(\mathbf{x}, t)+\boldsymbol{\nabla} \cdot[\mathbf{U}(\mathbf{x}) P(\mathbf{x}, t)-D \nabla P(\mathbf{x}, t)]=0,
$$

where $\mathbf{x}=(x, y)$ is the position vector in the $2 \mathrm{D}$ microdevice, $\mathbf{U}(\mathbf{x})$ is the velocity of the suspended particles, and
PACS numbers: 05.40.- a, 05.10.Gg, 47.61.- $\mathrm{k}, 47.85 . \mathrm{Np}$

$D$ is the diffusion coefficient [11]. This equation is completely analogous to the convection-diffusion equation for a transported solute [12], and the no-flux boundary condition is imposed at the surface of impermeable obstacles. In general, we are interested in computing the probability density flux describing the migration of the suspended particles, $\mathbf{J}(\mathbf{x}, t)=[\mathbf{U}(\mathbf{x}) P(\mathbf{x}, t)-D \boldsymbol{\nabla} P(\mathbf{x}, t)]$. In order to describe the evolution of the probability density in a periodic system, such as the one shown in Fig. 1, it is convenient to introduce the reduced probability density and flux [13] (a completely analogous approach using the convection-diffusion equation is developed in Ref. [12]),

$$
\begin{gathered}
\tilde{P}(\mathbf{x}, t)=\sum_{k_{x}=-\infty}^{+\infty} \sum_{k_{y}=-\infty}^{+\infty} P\left(x+k_{x} L_{x}, y+k_{y} L_{y}\right), \\
\tilde{\mathbf{J}}(\mathbf{x}, t)=\sum_{k_{x}=-\infty}^{+\infty} \sum_{k_{y}=-\infty}^{+\infty} \mathbf{J}\left(x+k_{x} L_{x}, y+k_{y} L_{y}\right),
\end{gathered}
$$

where $\mathbf{k}=\left(k_{x}, k_{y}\right)$ identifies a specific cell within the periodic system and $L_{x}, L_{y}$ are the unit cell dimensions (see Fig. 1). The previous sums are convergent, due to the normalization condition for $P(\mathbf{x}, t)$. Note that we have

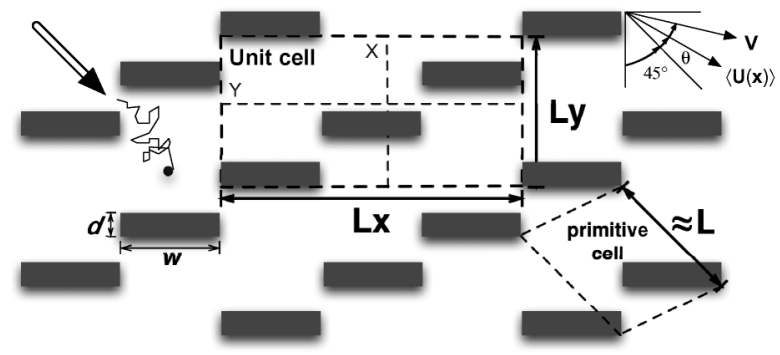

FIG. 1. Schematic view of the microfluidic device investigated here and in Refs. [4,6-8]. The gray rectangles are solid obstacles. Suspended particles are transported through the system by a velocity (or force) field. The unit cell used in this work and the primitive cell of the system are also shown. 
assumed an array of obstacles extending to infinity in both $x$ and $y$ directions, thus neglecting possible effects originating in the finite size of the device. Then, given the linearity of the Fokker-Planck equation, its invariance under discrete translations by $\left(L_{x}, L_{y}\right)$, and the convergence of the reduced probability density, it is clear that $\tilde{P}(\mathbf{x}, t)$ satisfies the Fokker-Planck equation within a unit cell, with periodic boundary conditions in the probability density and its flux. Therefore, for transit times longer than the diffusive time required for a single particle to explore the primitive unit cell, $\tau_{D} \approx L^{2} / D$, the probability distribution approaches the steady state solution of the FokkerPlanck equation. Note that only the reduced probability reaches a steady state, whereas the global probability density function has no meaningful asymptotic state [13]. The characteristic convective time for a particle moving through $N$ cells is $\tau_{C} \approx N L / U$, where $U$ is the magnitude of the coarse grained velocity of the particles, $\langle\mathbf{U}(\mathbf{x})\rangle$. Then, the asymptotic behavior corresponds to small global Peclet numbers, $\mathrm{Pe}=U L / N D \ll 1$. Typically, the steady state distribution is reached after the suspended particles are transported through a relatively small number of unit cells. In Ref. [8], for example, the authors observe lateral separation for $48.5 \mathrm{kbp}$ DNA molecules with $\mathrm{Pe} \approx 9 / \mathrm{N}$ $\left(U \sim 1 \mu \mathrm{m} ; D \sim 0.64 \mu \mathrm{m}^{2} / \mathrm{s} ; L \sim 6 \mu \mathrm{m}\right)$ but no lateral separation for 411 bp DNA molecules with $\mathrm{Pe} \approx 0.5 / \mathrm{N}$ $\left(U \sim 1 \mu \mathrm{m} / \mathrm{s}, \quad D \sim 12 \mu \mathrm{m}^{2} / \mathrm{s}\right.$ and $\left.L \sim 6 \mu \mathrm{m}\right)$. The steady state distribution is therefore reached at relatively small distances from the inlet (the total number of cells in the microdevice is $N_{T} \sim 1000$ ).

In the case of a mixture of different species, we can independently solve the Fokker-Planck equation for each of the species present in the system,

$$
\begin{array}{r}
\boldsymbol{\nabla} \cdot \tilde{\mathbf{J}}^{\infty}(\mathbf{x})=\boldsymbol{\nabla} \cdot\left[\mathbf{U}(\mathbf{x}) \tilde{P}^{\infty}(\mathbf{x})-D \boldsymbol{\nabla} \tilde{P}^{\infty}(\mathbf{x})\right]=0, \\
\left.\tilde{\mathbf{J}}^{\infty}(\mathbf{x}) \cdot \mathbf{n}(\mathbf{x})\right|_{\partial s}=0,
\end{array}
$$

where $\partial s$ is the surface of the obstacles and $\mathbf{n}$ is the normal to the solid surface. Then, we can determine the migration velocity, $\mathbf{V}$, by computing the total probability flux across the boundaries of the unit cell,

$$
V_{x}=L_{x} \int_{0}^{L_{y}} d y \tilde{J}_{x}^{\infty}
$$

and an analogous equation for $V_{y}$. We define the migration angle $\Delta \theta$ as the angle between $\mathbf{V}$ and $\langle\mathbf{U}(\mathbf{x})\rangle$ (see Fig. 1 and Ref. [12]).

We investigate the behavior of tracer particles driven either by an electrophoretic force, a gravity (centrifugal) force, or a flow field. In the first two cases the driving force is balanced by the viscous drag acting on the particles and the resulting velocities are proportional to the force fields, $\mathbf{U}=\eta_{h} \mathbf{F}$ or $\mathbf{U}=\eta_{\mathrm{ep}} \mathbf{E}$, where $\mathbf{F}, \mathbf{E}$ are the gravity (centrifugal) and electric fields, and $\eta_{h}, \eta_{\mathrm{ep}}$ are the hydrodynamic and electrophoretic mobilities, respectively [14]. In the case of particles convected by a pressure driven flow, the velocity of the tracer is assumed equal to the flow velocity. Let us now classify the velocity (or force) fields into permeating and nonpermeating fields, corresponding to nonzero and zero normal component at the surface of the solid obstacles, respectively.

Case I: Solenoidal, nonpermeating fields. - This is the case of electrophoretic fields and pressure driven flows. That is, if the obstacles are not permeable to the electric or flow fields, then the normal velocity is zero at the solid boundaries. In addition, both the flow field for incompressible fluids and the electric field have zero divergence. (Note that the electric field is solenoidal and nonpermeating outside the electrical double layer, which is typically a few nanometers thick, or in the absence of distributed charges.) In the absence of a driving field normal to the obstacles the no-flux boundary condition corresponds to zero probability (concentration) gradients normal to the solid-liquid interfaces. In fact, the steady state solution in this case corresponds to a uniform concentration, independent of the details of the driving field or the physical properties of the suspended species. Therefore, vector separation is not possible, given that the migration angle of any species is the same, with $\mathbf{V}=\langle\mathbf{U}(\mathbf{x})\rangle$. That is, if the magnitude of the local velocity of the particles is different for different species $\alpha$ and $\beta$, e.g., $\eta_{\mathrm{ep}}^{\alpha} \neq \eta_{\mathrm{ep}}^{\beta}$, then the particles would move at different speeds, but in the same direction, $\mathbf{V}^{\alpha}=\left(\eta_{\mathrm{ep}}^{\alpha} / \eta_{\mathrm{ep}}^{\beta}\right) \mathbf{V}^{\beta}$.

Case II: Permeating fields. - This is the case of gravity or centrifugal force fields. In Fig. 2 we show a contour plot of $\tilde{P}^{\infty}(\mathbf{x})$ for a spatially uniform force oriented at $45^{\circ}$ $\left(F_{x}=F_{y}\right)$. It is clear that concentration gradients develop close to the solid obstacles, which increase with the magnitude of the external force $F$. Furthermore, writing Eqs. (4) in dimensionless form shows that, for a given force field, the steady state probability distribution depends only on the local Peclet number, $\mathrm{Pe}_{\ell}=U d / D$, where $d$ is a characteristic length scale for the obstacles (see Fig. 1). Therefore, vector separation is possible for a mixture of
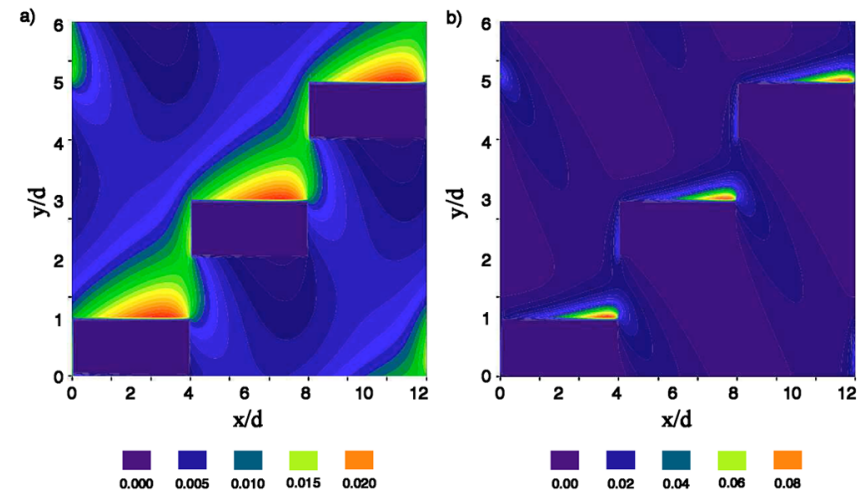

FIG. 2 (color online). Contour plots for the steady state reduced probability density function $\tilde{P}^{\infty}(\mathbf{x})$ inside a unit cell. The external force is spatially uniform and oriented at $45^{\circ}, F_{x}=F_{y}$. The plots correspond to (a) $\mathrm{Pe}_{\ell}=1.12$ and (b) $\mathrm{Pe}_{\ell}=5.58$. 
species with different local Peclet numbers. On the other hand, differences in the diffusivity of different species might not be enough to achieve separation if the local Peclet number of different species is the same (see example below). Figure 2 presents results for two different Peclet numbers, $\mathrm{Pe}_{\ell} \sim 1$ and $\mathrm{Pe}_{\ell} \sim 5$, which are representative of the experiments on the transport of DNA molecules in microdevices. For example, in Ref. [8] the authors report good separation in the range $2.1<\mathrm{Pe}_{\ell}<5.1$ and in Ref. [6] the authors observe the best separation performance for $\mathrm{Pe}_{\ell} \sim 3.5$. In general, the migration velocity of each species will depend on the permeating field. However, there will be no vector separation unless different species have different Peclet numbers. Consider, for example, a mixture of particles of different size driven through a stagnant fluid under the action of the same external force, F. Assuming that the local velocity of species $\alpha$ is determined by the hydrodynamic mobility of the particles, $\mathbf{U}^{\alpha}=\eta_{h}^{\alpha} \mathbf{F}$, and taking into account the Einstein relation, $D^{\alpha}=\eta_{h}^{\alpha} k_{B} T$, we obtain that all the species have the same Peclet number, $\mathrm{Pe}_{\ell}=U d / D=(F d) /\left(k_{B} T\right)$. Therefore, even though different particles have different diffusion coefficients they move in the same direction, and no vector separation is possible. On the other hand, if the same mixture of particles is driven by a centrifugal force, and assuming that they have the same density, they will move at different angles due to differences in the Peclet number, $\mathrm{Pe}_{\ell}^{\alpha}=\left(F^{\alpha} d\right) /(k T)$.

In order to test the previous results, we compared the predictions based on the steady state solution to Eqs. (4) with direct Monte Carlo simulations. The simulations were performed by solving the Langevin equation on the position of the particle, using periodic boundary conditions in both directions. Typically, we follow the trajectory of an individual particle for times $t \geq 10 \tau_{D}$, and we average the results over 1000 particles. First, we simulated the motion of tracer particles under the action of an electric, nonpermeating field and obtained, as predicted, a uniform distribution of particles. The computed migration velocity of the particles, $\mathbf{V}_{\mathrm{MC}} / U_{S}=(0.93 \pm 0.10,0.56 \pm 0.09)$, also agrees with that calculated from Eq. (5), $\mathbf{V}_{\mathrm{FP}} / U_{S}=$ $(0.94,0.58)$, where $U_{S}$ is the Stokes velocity in the absence of obstacles. Then, we simulated the motion of tracer particles under the action of a spatially uniform force. In Fig. 3 we compare the steady state solution presented in Fig. 2 with the simulation results along two cross sections of the unit cell, with excellent agreement. We also compare the asymptotic distribution of two species that experience the same force but have diffusivities that differ by a factor of 10. In agreement with our previous analysis, the asymptotic distributions are the same, independent of the diffusivity of the particles. In Fig. 4 we present the migration angle of the particles as a function of the local Peclet number, and show excellent agreement with the angles computed from Eqs. (5). Then, Figs. 3 and 4 support the conclusion that vector separation is possible if, and only if,

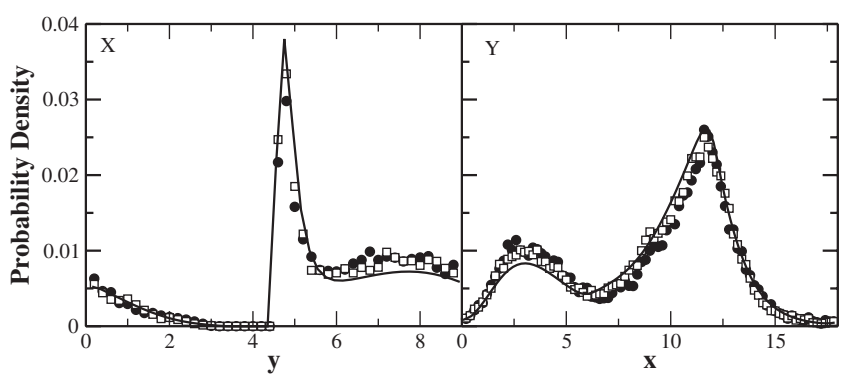

FIG. 3. Probability distribution function in the unit cell, at lines $X$ and $Y$ shown in Fig. 1. The force is spatially uniform and oriented at $45^{\circ} . \mathrm{Pe}_{\ell}=5.58$. The solid lines correspond to the solution to Eqs. (4) presented in Fig. 2(b). The open and solid points correspond to Monte Carlo simulations for two types of particles, which only differ on their mobility, and thus their diffusion coefficient, by a factor of 10 .

different particles have different $\mathrm{Pe}_{\ell}$. On the other hand, the previous results do not explain the separation observed in experiments with nonpermeating fields [6-8].

A possible source for the discrepancy between the previous analysis and the experimental observations is the finite size of the suspended particles [8]. To investigate finite-size effects we performed similar Monte Carlo simulations but accounting for excluded volume effects. That is, we include a hard-sphere potential that prevents finite-size particles from overlapping with the obstacles (in this approximation we neglect hydrodynamic and electrostatic interactions between the particles and the solid obstacles).

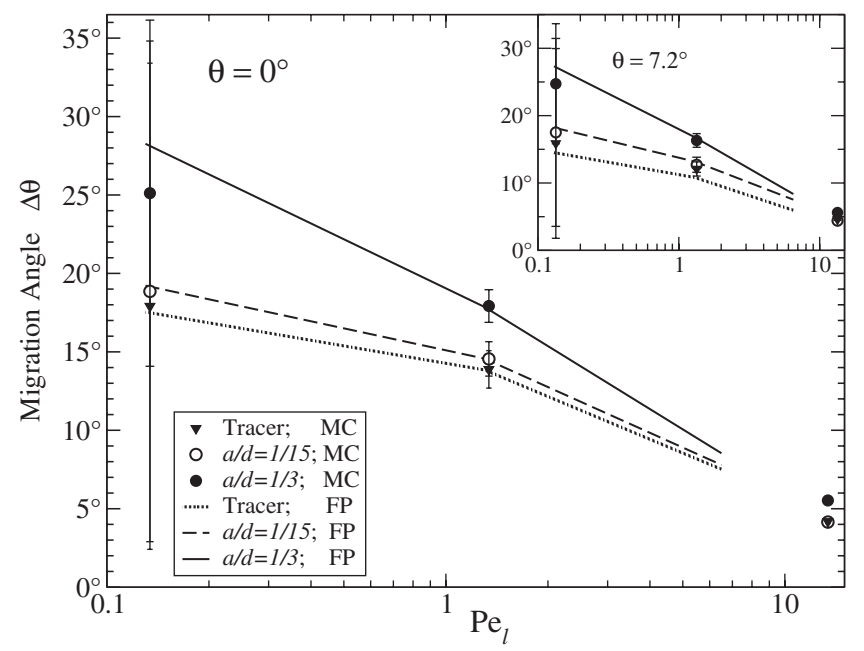

FIG. 4. Migration angle as a function of local Peclet number in a spatially uniform (permeating) field. The results correspond to tracer particles and finite-size particles $(a / d=1 / 3$ and $a / d=$ $1 / 15$, where $a$ is the particle radius) for two different tilting angles $\left(\theta=0^{\circ}\right.$ and $\left.\theta=7.2^{\circ}\right)$. The definitions of the tilt and migration angles are shown in Fig. 1. Symbols correspond to Monte Carlo simulations and lines correspond to predicted values from Eqs. (5) (note that due to the high computational cost of solving convection-diffusion equations at high $\mathrm{Pe}_{\ell}$ we were limited to $\left.\mathrm{Pe}_{\ell} \lesssim 5[15]\right)$. 


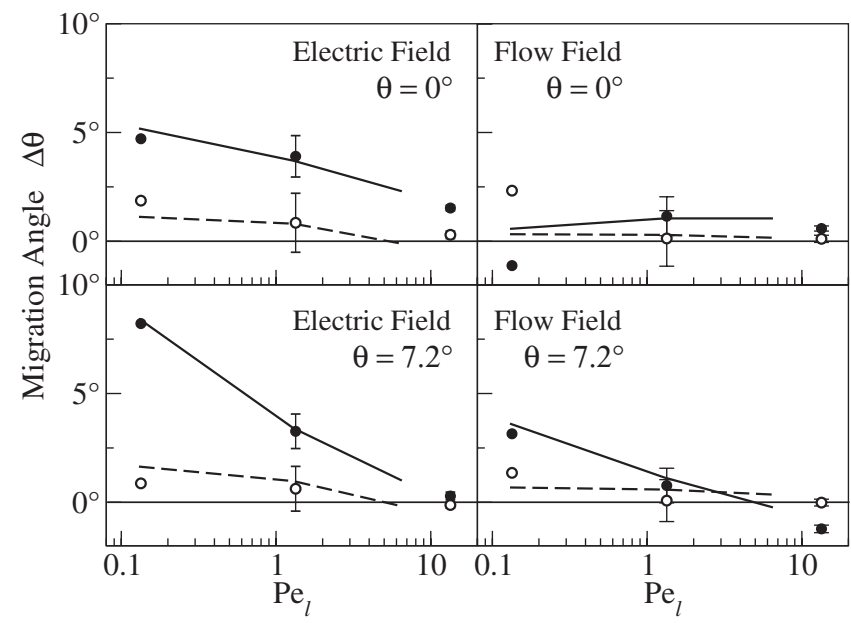

FIG. 5. Migration angle as a function of the local Peclet number for finite-size particles. The results are analogous to those presented in Fig. 4 but for an electrostatic field (left) and a pressure driven flow (right).

The migration angles for tracer and finite-size particles in the permeating, uniform field are shown in Fig. 4. A small enhancement is observed for large particles, $a / d=1 / 3$, where $a$ is the radius of the particles. More important, in Fig. 5, we show that the finite size of the particles induces nonzero migration angles, especially in the case of electrophoretic forces and relatively large particles, $a / d=1 / 3$. The largest migration angles are obtained at low $\mathrm{Pe}_{\ell}, \mathrm{a}$ regime that, on the other hand, has low resolution due to diffusive broadening. Similar results are observed for a different tilt angle, $\theta=7.2^{\circ}$, which is the tilt angle showing the largest lateral migration in Ref. [7]. The migration angles are generally small and similar to those reported in the experiments, including negative migration angles for large $\mathrm{Pe}_{\ell}$ (not shown in the figures). However, a direct comparison is not possible due to the large size of the DNA molecules $(a / d \sim 0.37$ and $a / d \sim 0.68$, which is larger than the gaps between the obstacles) and the high local Peclet numbers used in the experiments $\left(\mathrm{Pe}_{\ell} \sim 10-100\right)$. (Note that the limit of high Peclet numbers is singular, with high concentration gradients present in a thin boundary layer of thickness $\sim d / \mathrm{Pe}_{\ell}$, which for $d \approx 1 \mu \mathrm{m}$ and $\mathrm{Pe}_{\ell} \gtrsim 10$ becomes smaller than $\leqslant 100 \mathrm{~nm}$, and both hydrodynamic and electrostatic forces are likely to play a significant role.) Finally, we computed the migration angles of finite-size particles by means of Eq. (5). First, we exclude the region around the obstacles that is not accessible to finite-size particles, creating a shell of size $a$ surrounding the obstacles. We then solve the Fokker-Planck equation with new boundary conditions corresponding to the obstacles with their shells. The migration angles are then calculated as in the case of tracer particles, with excellent agreement (see Figs. 4 and 5).

In all cases, the migration angles observed in nonpermeating fields are substantially smaller than those obtained with spatially uniform, permeating fields. These results provide guidance for future developments in the design of microfluidic devices for separation. In the case of DNA molecules, for example, although the electrophoretic velocity is independent of size, the diffusivity, and thus the local Peclet number, is not. Therefore, it could be possible to achieve vector separation by creating an electric field with a nonvanishing normal component at the solid surfaces using, for example, permeable obstacles. The numerical solution of the Fokker-Planck equation in different geometries could then be used to optimize the design of such microdevices. Finally, we mention that further work is required to investigate the effect of particle size. Hydrodynamic and electrostatic interactions between the suspended particles and the solid obstacles also need to be considered for particle sizes comparable to the size of the obstacles, $a / d \gtrsim 0.5$, and for high local Peclet numbers.

This research used resources of the National Energy Research Scientific Computing Center, which is supported by the Office of Science of the U. S. Department of Energy under Contract No. DE-AC02- 05CH11231.

*Electronic address: zli@jhu.edu

†Electronic address: drazer@jhu.edu

[1] T. M. Squires and S. R. Quake, Rev. Mod. Phys. 77, 977 (2005).

[2] P.S. Dittrich, K. Tachikawa, and A. Manz, Anal. Chem. 78, 3887 (2006).

[3] J. Rousselet, L. Salome, A. Ajdari, and J. Prost, Nature (London) 370, 446 (1994).

[4] T. A. J. Duke and R. H. Austin, Phys. Rev. Lett. 80, 1552 (1998).

[5] D. Ertas, Phys. Rev. Lett. 80, 1548 (1998).

[6] C. F. Chou, O. Bakajin, S. W. P. Turner, T. A. J. Duke, S. S. Chan, E. C. Cox, H. G. Craighead, and R. H. Austin, Proc. Natl. Acad. Sci. U.S.A. 96, 13762 (1999).

[7] L. R. Huang, E. C. Cox, R. H. Austin, and J. C. Sturm, Anal. Chem. 75, 6963 (2003).

[8] L. R. Huang, P. Silberzan, J. O. Tegenfeldt, E. C. Cox, J. C. Sturm, R. H. Austin, and H. Craighead, Phys. Rev. Lett. 89, 178301 (2002).

[9] R. H. Austin, N. Darnton, R. Huang, J. Sturm, O. Bakajin, and T. Duke, Appl. Phys. A 75, 279 (2002).

[10] K. D. Dorfman and H. Brenner, Phys. Rev. E 65, 052103 (2002).

[11] H. Risken, The Fokker-Planck Equation, Methods of Solution and Application (Springer, Berlin, 1989), 2nd ed.

[12] H. Brenner and D. A. Edwards, Macrotransport Processes (Butterworth-Heinemann, Boston, 1993).

[13] P. Reimann, Phys. Rep. 361, 57 (2002).

[14] W. B. Russel, D. A. Saville, and W. R. Schowalter, Colloidal Dispersions (Cambridge University Press, Cambridge, England, 1989).

[15] J. J. Kirchner and E. F. Hasselbrink, Jr., Anal. Chem. 77, 1140 (2005). 